\title{
Inverse Eigenvalue Problem for Generalized Arrow-Like Matrices
}

\author{
Zhibin Li, Cong Bu, Hui Wang \\ College of Mathematics, Dalian Jiaotong University, Dalian, China \\ E-mail: lizhibinky@163.com \\ Received October 14, 2011; revised November 15, 2011; accepted November 23, 2011
}

\begin{abstract}
This paper researches the following inverse eigenvalue problem for arrow-like matrices. Give two characteristic pairs, get a generalized arrow-like matrix, let the two characteristic pairs are the characteristic pairs of this generalized arrow-like matrix. The expression and an algorithm of the solution of the problem is given, and a numerical example is provided.
\end{abstract}

Keywords: Generalized Arrow-Like Matrices, Characteristic Value, Inverse Problem, Unique

\section{Introduction}

The Inverse eigenvalue problem for matrices in the problems involved in the field of structural design, pattern recognition, parameter recognition, automatic control and so on, it has a good engineering background, and its research has obvious significance [1]. Many experts and scholars have addressed more extensively and in-depth studied, get a lot of conclusions about inverse eigenvalue problem for Jacobi matrices [2], but there is less research about the inverse eigenvalue problem for arrow- like matrices $[3,4]$. This paper researches the following inverse eigenvalue problem for generalized arrow-like matrices.

Generalized arrow-like matrices refer to the matrix as follows:

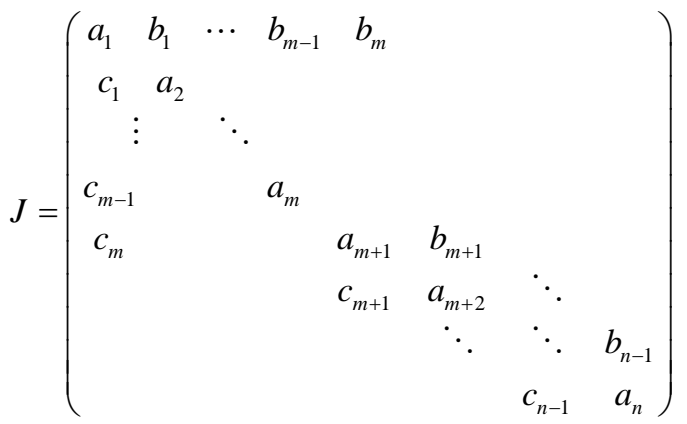

When $m=1, J$ becomes generalized Jacobi matrix [1]; when $m=n, J$ is an arrow-like matrice. This article studies the following characteristic value inverse:

Question IEPGAM. Given two real numbers

$\lambda, \mu(\lambda \neq \mu)$ and two nonzero real vectors $x=\left(x_{1}, x_{2}, \cdots, x_{n}\right)^{\mathrm{T}} \in R^{n}, y=\left(y_{1}, y_{2}, \cdots, y_{n}\right)^{\mathrm{T}} \in R^{n}$.

Find the $n \times n$ real generalized arrow-like matrice $J$, such that $J x=\lambda x, J y=\mu y$.

The expression and an algorithm of the solution of the problem is given in Section 2, and a numerical example is provided in Section 3 .

\section{The Solution of Question IEPGAM}

Because $(\lambda, x)$ and $(\mu, y)$ are two characteristic pairs of the generalized arrow-like matrices $J$,

In it,

Let:

$$
\begin{aligned}
x_{0}=x_{n+1}=y_{0} & =y_{n+1}=b_{0}=b_{n}=c_{0}=c_{n}=0, \\
D_{i} & =\left|\begin{array}{cc}
x_{i} & y_{i} \\
x_{i+1} & y_{i+1}
\end{array}\right|(i=0,1, \cdots, n), \\
E_{i} & =\left|\begin{array}{ll}
x_{1} & x_{i} \\
y_{1} & y_{i}
\end{array}\right|(i=2,3, \cdots, m+1)
\end{aligned}
$$

So

$$
\begin{gathered}
a_{1} x_{1}+b_{1} x_{2}+\cdots+b_{m-1} x_{m}+b_{m} x_{m+1}=\lambda x_{1}, \\
c_{1} x_{1}+a_{2} x_{2}=\lambda x_{2}, \\
\cdots \\
c_{m-1} x_{1}+a_{m} x_{m}=\lambda x_{m}, \\
c_{m} x_{1}+a_{m+1} x_{m+1}+b_{m+1} x_{m+2}=\lambda x_{m+1}, \\
c_{m+1} x_{m+1}+a_{m+2} x_{m+2}+b_{m+2} x_{m+3}=\lambda x_{m+2},
\end{gathered}
$$




$$
\begin{gathered}
c_{n-2} x_{n-2}+a_{n-1} x_{n-1}+b_{n-1} x_{n}=\lambda x_{n-1}, \\
c_{n-1} x_{n-1}+a_{n} x_{n}=\lambda x_{n} . \\
a_{1} y_{1}+b_{1} y_{2}+\cdots+b_{m-1} y_{m}+b_{m} y_{m+1}=\mu y_{1},(6-1) \\
c_{1} y_{1}+a_{2} y_{2}=\mu y_{2}, \\
\ldots \\
c_{m-1} y_{1}+a_{m} y_{m}=\mu y_{m}, \\
c_{m} y_{1}+a_{m+1} y_{m+1}+b_{m+1} y_{m+2}=\mu y_{m+1}, \\
c_{m+1} y_{m+1}+a_{m+2} y_{m+2}+b_{m+2} y_{m+3}=\mu y_{m+2}, \\
\ldots \\
c_{n-2} y_{n-2}+a_{n-1} y_{n-1}+b_{n-1} y_{n}=\mu y_{n-1}, \\
c_{n-1} y_{n-1}+a_{n} y_{n}=\mu y_{n} .
\end{gathered}
$$

- For inverse $b_{i}, c_{i}(i=m+1, m+2, \cdots, n-1)$, $a_{i}(i=m+2, m+3, \cdots, n)$.

From (5) and (6), we can get

$$
\begin{aligned}
c_{i-1} x_{i-1}+a_{i} x_{i}+b_{i} x_{i+1} & =\lambda x_{i}(i=m+2, m+3, \cdots, n), \\
c_{i-1} y_{i-1}+a_{i} y_{i}+b_{i} y_{i+1} & =\mu y_{i}(i=m+2, m+3, \cdots, n) .
\end{aligned}
$$

In order to eliminate $a_{i}$, multiply by $y_{i}$ on both sides of (7), multiply by $x_{i}$ on both sides of (8), then cut on both sides, we can get

$$
b_{i} D_{\mathrm{i}}=(\mu-\lambda) x_{i} y_{i}+c_{i-1} D_{i-1}(i=m+2, m+3, \cdots, n) .
$$

To problem $A$, because $c_{i}=k b_{i},(i=2,3, \cdots, n-1)$, so (9) become

$$
\begin{aligned}
b_{i} D_{i} & =(\mu-\lambda) x_{i} y_{i}+k b_{i-1} D_{i-1} \\
(i & =m+2, m+3, \cdots, n)
\end{aligned}
$$

Let $i=n$, because $D_{n}=0$, so

$$
b_{n-1} D_{n-1}=\frac{x_{\mathrm{n}} y_{n}}{k}(\lambda-\mu),
$$

Let $i=n-1, \quad b_{n-2} D_{n-2}=(\lambda-\mu)\left[\frac{x_{n} y_{n}}{k^{2}}+\frac{x_{n-1} y_{n-1}}{k}\right]$;

Let $i=m+2$,

$b_{m+1} D_{m+1}=(\lambda-\mu)\left[\frac{x_{n} y_{n}}{k^{n-(m+1)}}+\frac{x_{n-1} y_{n-1}}{k^{n-(m+2)}}+\cdots+\frac{x_{m+2} y_{m+2}}{k}\right]$

Under normal circumstances,

$b_{j} D_{j}=(\lambda-\mu) \sum_{s=0}^{n-(j+1)} \frac{x_{n-s} y_{n-s}}{k^{n-(s+j)}}(j=m+1, m+2, \cdots, n-1)$.

If $D_{j} \neq 0(j=m+1, m+2, \cdots, n-1)$, then $x_{i}, y_{i}$ can not be zero at the same time, so

$$
b_{j}=\frac{(\lambda-\mu)}{D_{j}} \sum_{s=0}^{n-(j+1)} \frac{x_{n-s} y_{n-s}}{k^{n-(s+j)}}(j=m+1, m+2, \cdots, n-1),
$$

$$
\begin{aligned}
& c_{j}=k b_{j},(j=m+1, m+2, \cdots, n-1), \\
& a_{j}=\left\{\begin{array}{l}
\frac{\lambda x_{j}-c_{j-1} x_{j-1}-b_{j} x_{j+1}}{x_{j}}, x_{j} \neq 0 ; \\
\frac{\mu y_{j}-c_{j-1} y_{j-1}-b_{j} y_{j+1}}{y_{j}}, y_{j} \neq 0
\end{array}\right. \\
& (j=m+2, m+3, \cdots, n)
\end{aligned}
$$

- For inverse $a_{m+1}, c_{m}, b_{m}$.

From (5) and the $m+1$ equation of (6),

$$
\begin{gathered}
c_{m} E_{m+1}=(\lambda-\mu) x_{m+1} y_{m+1}+b_{m+1} D_{m+1}, \\
a_{m+1} E_{\mathrm{m}+1}=\mu x_{1} y_{m+1}-\lambda x_{m+1} y_{1}-b_{m+1} E_{\mathrm{m}+2}, \\
b_{m}=\frac{c_{m}}{k} .
\end{gathered}
$$

- For inverse $c_{1}, c_{i}, b_{i}(i=2,3, \cdots, m-1)$, $a_{i}(i=2,3, \cdots, m)$.

From (5) and 2 to $m$ equation of (6),

$$
\begin{gathered}
c_{i-1} x_{1}+a_{i} x_{i}=\lambda x_{i}(i=2,3, \cdots, m), \\
c_{i-1} y_{1}+a_{i} y_{i}=\mu y_{i}(i=2,3, \cdots, m) .
\end{gathered}
$$

From (18) and (19), we can get

$$
\begin{gathered}
c_{i-1} E_{\mathrm{i}}=(\lambda-\mu) x_{i} y_{i}(i=2,3, \cdots, m), \\
a_{i} E_{\mathrm{i}}=\mu x_{1} y_{i}-\lambda x_{i} y_{1}(i=2,3, \cdots, m), \\
b_{i}=\frac{c_{i}}{k}(i=2,3, \cdots, m-1) .
\end{gathered}
$$

- For inverse $a_{1}, b_{1}$.

From (5) and (6), we can get

$$
\begin{aligned}
& a_{1} x_{1}+b_{1} x_{2}=\lambda x_{1}-\sum_{s=2}^{m} b_{s} x_{s+1}, \\
& a_{1} y_{1}+b_{1} y_{2}=\mu y_{1}-\sum_{s=2}^{m} b_{s} y_{s+1} .
\end{aligned}
$$

If $D_{1} \neq 0$, from (23) and (24), then we can get

$$
\begin{gathered}
a_{1}=\frac{\lambda x_{1} y_{2}-\mu x_{2} y_{1}-\sum_{s=2}^{m} b_{s}\left(x_{s+1} y_{2}-x_{2} y_{s+1}\right)}{D_{1}}, \\
b_{1}=\frac{(\mu-\lambda) x_{1} y_{1}-\sum_{s=2}^{m} b_{s}\left(y_{s+1} x_{1}-y_{1} x_{s+1}\right)}{D_{1}} .
\end{gathered}
$$

According to the above analysis, to question IEPGAM, we can get the follow theorem.

Theorem. If the following conditions are satisfied:

1) $D_{1} \neq 0$;

2) $D_{i} \neq 0(i=m+1, m+2, \cdots, n-1)$;

3) $E_{i} \neq 0(i=2,3, \cdots, m+1)$

Then question IEPGAM has the unique solution, and 
$b_{j}=\frac{(\lambda-\mu)}{D_{j}} \sum_{s=0}^{n-(j+1)} \frac{x_{n-s} y_{n-s}}{k^{n-(s+j)}}(j=m+1, m+2, \cdots, n-1)$

$$
\begin{aligned}
& a_{j}=\left\{\begin{array}{l}
\frac{\lambda x_{j}-c_{j-1} x_{j-1}-b_{j} x_{j+1}}{x_{j}}, x_{j} \neq 0 ; \\
\frac{\mu y_{j}-c_{j-1} y_{j-1}-b_{j} y_{j+1}}{y_{j}}, y_{j} \neq 0
\end{array},\right. \\
& (j=m+2, m+3, \cdots, n) \\
& b_{m}=\frac{(\lambda-\mu) x_{m+1} y_{m+1}+b_{m+1} D_{m+1}}{k E_{m+1}}, \\
& a_{m+1}=\frac{\mu x_{1} y_{m+1}-\lambda x_{m+1} y_{1}-b_{m+1} E_{\mathrm{m}+2}}{E_{m+1}}, \\
& b_{j}=\frac{(\lambda-\mu) x_{j+1} y_{j+1}}{k E_{j+1}}(i=2,3, \cdots, m-1), \\
& b_{1}=\frac{(\mu-\lambda) x_{1} y_{1}-\sum_{s=2}^{m} b_{s}\left(y_{s+1} x_{1}-y_{1} x_{s+1}\right)}{D_{1}} \\
& a_{j}=\frac{\mu x_{1} y_{j}-\lambda x_{j} y_{1}}{E_{j}}(j=2,3, \cdots, m) \text {, } \\
& a_{1}=\left\{\begin{array}{c}
\lambda-\frac{\sum_{s=1}^{m} b_{s} x_{s+1}}{x_{1}}, x_{1} \neq 0 ; \\
\mu-\frac{\sum_{s=1}^{m} b_{s} y_{s+1}}{y_{1}}, y_{j} \neq 0
\end{array}\right. \\
& c_{j}=k b_{j},(i=2,3, \cdots, n-1) \text {, } \\
& c_{1}=\frac{(\lambda-\mu) x_{2} y_{2}}{E_{2}} \text {. }
\end{aligned}
$$

\section{Numerical Examples}

Example 1. Give $\lambda=1, \mu=2, k=2, m=2, n=5$, $x=(1,1,1,1,1)^{\mathrm{T}}, y=(1,0,2,-1,0)^{\mathrm{T}}$.

It is easy to be calculated

$$
\begin{gathered}
D_{1}=-1 \neq 0, D_{2}=-3 \neq 0, D_{4}=1 \neq 0 ; \\
E_{2}=-1 \neq 0, E_{3}=1 \neq 0, E_{4}=-2 .
\end{gathered}
$$

From Theorem, the question IEPGAM has the unique solution. And

$$
\begin{gathered}
b_{3}=\frac{\lambda-\mu}{D_{3}}\left[\frac{x_{5} y_{5}}{k^{2}}+\frac{x_{4} y_{4}}{k}\right]=-\frac{1}{6}, \\
b_{2}=\frac{1}{k E_{3}}\left[(\lambda-\mu) x_{3} y_{3}+b_{3} D_{3}\right]=-\frac{3}{4}, \\
b_{4}=\frac{\lambda-\mu}{D_{4}}\left[\frac{x_{5} y_{5}}{k}\right]=0,
\end{gathered}
$$

$$
\begin{gathered}
b_{1}=\frac{(\mu-\lambda) x_{1} y_{1}-b_{2}\left(y_{3} x_{1}-y_{1} x_{3}\right)}{D_{1}}=-\frac{7}{4} ; \\
c_{2}=k b_{2}=-\frac{3}{2}, \\
c_{3}=k b_{3}=-\frac{1}{3}, \\
c_{4}=k b_{4}=0, \\
c_{1}=\frac{(\lambda-\mu) x_{2} y_{2}}{E_{2}}=0 ; \\
a_{1}=\lambda-\frac{b_{1} x_{2}+b_{2} x_{3}}{x_{1}}=\frac{7}{2}, \\
a_{2}=\frac{\mu x_{1} y_{2}-\lambda x_{2} y_{1}}{E_{2}}=1, \\
a_{3}=\frac{\mu x_{1} y_{3}-\lambda x_{3} y_{1}-b_{3} E_{4}}{E_{3}}=\frac{8}{3}, \\
a_{4}=\frac{\lambda x_{4}-c_{3} x_{3}-b_{4} x_{5}}{x_{4}}=\frac{4}{3}, \\
a_{5}=\frac{\lambda x_{5}-c_{4} x_{4}-b_{5} x_{6}}{x_{5}}=1 .
\end{gathered}
$$

So

and $J x=\lambda x, J y=\mu y$.

$$
J=\left(\begin{array}{ccccc}
\frac{7}{2} & \frac{-7}{4} & \frac{-3}{4} & 0 & 0 \\
0 & 1 & 0 & 0 & 0 \\
\frac{-3}{2} & 0 & \frac{8}{3} & \frac{-1}{6} & 0 \\
0 & 0 & \frac{-1}{3} & \frac{4}{3} & 0 \\
0 & 0 & 0 & 0 & 1
\end{array}\right)
$$

\section{References}

[1] D. J. Wang, "Inverse Eigenvalue Problem in Structural Dynamics,” Journal of Vibration and Shock, No. 2, 1988, pp. 31-43.

[2] H. Dai, "Inverse Eigenvalue Problem for Jacobi Matrices,” Computation Physics, Vol. 11, No. 4, 1994, pp. 451-456.

[3] C. H. Wu and L. Z. Lu, "Inverse Eigenvalue Problem for a Kind of Special Matrix,” Journal of Xiamen University (Natural Science), No. 1, 2009, pp. 22-26.

[4] Q. X. Yin, "Generalized Inverse Eigenvalue Problem for Arrow-Like Matrices,” Journal of Nan Jing University of Aeronautics \& Astronautics, Vol. 34, No. 2, 2002, pp. 190-192. 